\title{
芳香磺酰肼与胺通过铜催化氧化偶联合成芳香磺酰胺
}

\author{
张振雷* 钱 朋 查正根* \\ (中国科学技术大学化学与材料学院 合肥 230026)
}

\begin{abstract}
摘要 报道了在铜催化的条件下, 芳香磺酰肼与各种胺氧化偶联合成芳香磺酰胺化合物. 相比于传统的合成磺酰胺方 法, 该合成方法利用铜和氧气作为氧化体系, 无需额外添加其他氧化剂, 副产物只有氮气, 是一个比较绿色的合成磺 酰胺类化合物的方法.
\end{abstract}

关键词＼cjkstart铜催化; 磺酰肼; 磺酰胺

\section{Copper-Catalyzed Aerobic Oxidative Coupling of Aromatic Sulfonyl Hydrazides with Amines: A New Access to Aromatic Sulfonamides}

\author{
Zhang, Zhenlei* Qian, Peng ${ }^{a} \quad$ Zha, Zhenggen* \\ (School of Chemistry and Materials Science, University of Science and Technology of China, Hefei 230026)
}

\begin{abstract}
A copper(II)-catalyzed aerobic oxidative coupling of aromatic sulfonyl hydrazides with amines for the synthesis of aromatic sulfonamides was described. In contrast to previously described methods, this reaction employs copper/ $\mathrm{O}_{2}$ as the catalytic system, and generates $\mathrm{N}_{2}$ as the only byproduct, which provides an environmentally benign synthetic route for aromatic sulfonamides.
\end{abstract}

Keywords copper catalysis; sulfonyl hydrazide; sulfonamide

\section{Introduction}

Free radicals could enable reactions that would be difficult to achieve with typical ionic transformations. ${ }^{[1]}$ Therefore, the invention of powerful catalytic radicalbased reactions for the selective formation of chemical bonds has attracted considerable attention from synthetic chemists. In particular, studies on $\mathrm{Cu}(\mathrm{II}) / \mathrm{O}_{2}$ catalytic system have demonstrated its abilities to not only transfer one electron to the corresponding substrate but also to be regenerated by aerobic oxidation of the $\mathrm{Cu}(\mathrm{I})$ species, which allow the design of unique single electron transfer pathways that enable the development of many environmentally benign chemical transformations. ${ }^{[2]} \mathrm{Up}$ to date, success in this area of catalysis is still limited mostly to the construction of carbon-carbon and carbon-heteroatom bonds, ${ }^{[3]}$ while fragment coupling methods under $\mathrm{Cu}(\mathrm{II}) / \mathrm{O}_{2}$ catalytic system that construct hetero-heteroatom bonds are relatively limited ${ }^{[4]}$ despite the opportunity to access valuable structural motifs of biologically active molecules. Sulfonamides are an important class of structural moieties widely present in natural products and pharmaceuticals, such as<smiles>Nc1ccc(S(=O)(=O)N(Cc2ccccc2)CC(O)C(Br)NC(=O)OC2CCOC2)cc1</smiles><smiles>Cc1cc(C(F)(F)F)nn1-c1ccc(S(N)(=O)=O)cc1</smiles>

Figure 1 Drugs containing sulfonamide structure motif amprenavir and celecoxib (Figure 1). ${ }^{[5]}$ Given its importance, the preparation of sulfonamide unit has attracted much attention from the community. ${ }^{[6 \sim 17]}$ In general, sulfonamides can be prepared either by the straightforward reaction of amines with sulfonic acid derivatives ${ }^{[6]}$ or the alkylation of sulfonamides with organic halides ${ }^{[7]}$ pseudo halides ${ }^{[8]}$ or alcohol.$^{[9]}$ However, the challenges along with

* Corresponding authors. E-mail: helenken@mail.ustc.edu.cn; zgzha@ustc.edu.cn

Received March 5, 2019; revised March 26, 2019; published online April 19, 2019

Project supported by the National Natural Science Foundation of China (Nos. 21672200, 21472177)

国家自然科学基金(Nos. 21672200, 21472177)资助项目. 
sulfonamide synthesis originate mostly from the use of non-stable, hazardous starting materials, generating a large quantity of toxic byproduct, and the requirement of harsh conditions. To address this issue, many groups have reported the improved sulfonamide synthesis employing inexpensive catalyst, starting materials and the reduction of the amount of environmental unfriendly byproducts. For instance, Jiang and co-workers reported an efficient copper-catalyzed sulfonamide synthesis from sodium sulfinates and amines (Scheme 1a). ${ }^{[10]}$ However, $20 \mathrm{~mol} \%$ copper catalyst is needed. Sulfonamides synthesis starting from thios and sodium sulfinates mediated by metal or iodine reagents were also independently developed by Pan, ${ }^{[11]}$ Wang, ${ }^{[12]}$ Yuan $^{[13]}$ and Song ${ }^{[14]}$ groups (Scheme $1 \mathrm{~b} \sim 1 \mathrm{c})$. Recently, copper-catalyzed electrophilic amination of sodium sulfinates or aminosulfonylation of aryldiazonium tetrafluoroborates and $\mathrm{DABCO} \bullet\left(\mathrm{SO}_{2}\right)_{2}$ were also developed for the efficient synthesis of sulphonamides (Scheme 1d). ${ }^{[15]}$ Besides, $\mathrm{I}_{2} /$ peroxide or $\mathrm{I}_{2} \mathrm{O}_{5}$-mediated sulfonylation of amines also provided alternative approaches for sulfonamides (Scheme 1e). ${ }^{[16]}$ With the aim to synthesize biologically important molecules in an atom-economical manner, we believe that developing efficient and environmentally benign strategies for the construction of sulfonamide units via a radical process is still highly desired. By taking advantage of the stable and readily available nature of sulfonyl hydrazides, ${ }^{[18]}$ we herein report an aerobic oxidative synthesis of sulfonamides from sulfonyl hydrazines and amines with $\mathrm{N}_{2}$ as the only byproduct using $5 \mathrm{~mol} \%$ copper catalyst (Scheme 1f).

\section{Results and discussion}

Our investigation began with the reaction of sulfonyl hydrazine (1a) and piperidine (2a) (Table 1). Initially, a screening of the catalyst showed that $\mathrm{CuBr}_{2}$ was the best catalyst for this reaction (Entry 1), whereas other commonly used copper salts gave lower yields (Entries 2 5). When other Lewis acids, such as $\mathrm{FeCl}_{3}$ and $\mathrm{NiCl}_{2}$, were employed as catalysts, no corresponding product was obtained (Entries 6 7). Subsequently, a series of solvents were evaluated. The results suggested that $N, N$-dimethylacetamide (DMA) was the best solvent for this reaction (Entries 8 vs Entries 9 12). Control experiment showed that a higher yield was obtained when 3 equiv. of piperidine was employed (Entry 14). Decreasing the amount of solvent to $0.1 \mathrm{~mL}$ improved the yield to $84 \%$ (Entry 15 ). Further evaluation of the reaction conditions revealed that a higher reaction temperature gave a lower yield (Entry 16). Note that replacing the oxygen atmosphere to air led to a slightly lower yield (Entry 17).

Under the optimized reaction conditions, the substrate scope of sulfonyl hydrazine 1 was examined. As shown in Table 2, a variety of sulfonyl hydrazines were tolerated well to give sulfonamides 3aa $\mathbf{3 l a}$ in good to excellent yields. The electron-donating and electron-withdrawing (a) Copper-catalyzed amination of sodium sulfinates (Jiang's work)

$$
\mathrm{R}^{1}-\stackrel{\mathrm{O}}{\mathrm{S}}-\mathrm{ONa}+\mathrm{R}^{2} \mathrm{R}^{3} \mathrm{NH}_{2} \stackrel{\mathrm{Cu}(\mathrm{II})(20 \mathrm{~mol} \%)}{\stackrel{\mathrm{O}_{2} \text { or } \mathrm{DMSO}}{\longrightarrow}} \mathrm{R}^{1}-\underset{\|_{\mathrm{O}}^{\mathrm{S}}}{\|}-\mathrm{NR}^{2} \mathrm{R}^{3}
$$

(b) Copper-mediated sulfonamide synthesis (Pan's work)

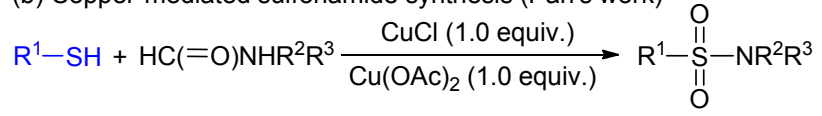

(c) lodine-mediated sulfonamide synthesis (Wang, Yuan and Song's work)

$\mathrm{R}^{1}-\stackrel{\mathrm{O}}{\mathrm{S}}-\mathrm{ONa}+\mathrm{R}^{2} \mathrm{R}^{3} \mathrm{NH} \stackrel{\mathrm{I}_{2}(0.5 \sim 1.0 \text { equiv. })}{\longrightarrow} \mathrm{R}^{1}-\stackrel{\mathrm{O}}{\|_{\mathrm{O}}}-\mathrm{NR}^{2} \mathrm{R}^{3}$

(d) Copper-catalyzed electrophilic amination (Tu and Wu's work)

$$
\begin{aligned}
& \mathrm{R}^{1} \mathrm{SO}_{2} \mathrm{Na} \text { or } \mathrm{R}^{1} \mathrm{~N}_{2} \mathrm{BF}_{4}+\mathrm{DABCO} \cdot\left(\mathrm{SO}_{2}\right)_{2}+\mathrm{R}^{2} \mathrm{R}^{3} \mathrm{NX} \stackrel{\mathrm{Cu}(\mathrm{II})}{\longrightarrow} \\
& \mathrm{R}^{1}-\mathrm{II}_{\mathrm{O}}^{\mathrm{O}}-\mathrm{NR}^{2} \mathrm{R}^{3} \quad \mathrm{X}=\mathrm{BzO}, \mathrm{Cl}
\end{aligned}
$$

(e) $\mathrm{I}_{2} /$ TBHP or $\mathrm{I}_{2} \mathrm{O}_{5}$ mediated oxidative sulfonamide synthesis

$$
\begin{aligned}
& \mathrm{R}^{1} \mathrm{SH} \text { or } \mathrm{R}^{1}-\mathrm{SO}_{2} \mathrm{NHNH}_{2}+\mathrm{R}^{2} \mathrm{R}^{3} \mathrm{NH}_{2} \underset{\text { or } \mathrm{I}_{2} \mathrm{O}_{5} \text { (1 equiv.) }}{\stackrel{\mathrm{I}_{2} / \mathrm{TBHP}}{ }} \\
& \mathrm{R}^{1}-\mathrm{I}_{\mathrm{O}}^{\mathrm{O}}-\mathrm{NR}^{2} \mathrm{R}^{3}
\end{aligned}
$$

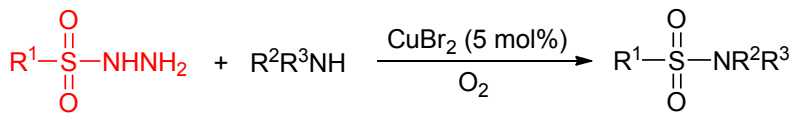

Scheme 1 Representive synthetic routes for the construction of sulfonamides

groups, such as methyl and halides, on the aromatic ring led to slightly lower yields (3aa $\sim 3 \mathbf{b a}, \mathbf{3 d a} \sim \mathbf{3 f a})$; However, the introduction of a strong electron-donating or electron-withdrawing group on the aromatic ring resulted in a decreased yield to $61 \%$ and $46 \%$, respectively (3ca and 3ga). Compared with substrates with para-substituents, those with ortho- and meta-substituents on the aromatic ring gave slightly lower yields $(\mathbf{3 h a} \sim \mathbf{3 j a})$. Moreover, the bulky substrate $\mathbf{1 j a}$ also underwent the reaction smoothly to give the corresponding sulfonamide $3 \mathbf{j a}$ in $75 \%$ yield. To our delight, when heteroarene sulfonyl hydrazide was employed as the substrate, the desired sulfonamide 3la could also be obtained in 59\% yield.

Next, we set out to survey the reactivity of diverse amines for the transformations, and the results were summarized in Table 3. Initially, various six-membered cyclic amines were tested under the optimized conditions. The results showed that the steric hindrance had a profound effect (3ad vs 3ab $\sim \mathbf{3 a c}$ ), while electronic effect of the substituent had little effect on the yields (3ae $\mathbf{3 a f})$. Next, the substrate scope of five-membered cyclic amines was also investigated, providing the desired products $\mathbf{3 a g}$ and 3ah in $74 \%$ and $62 \%$ yields, respectively. Interestingly, 
Table 1 Optimization of the Reaction Conditions ${ }^{a}$<smiles>Cc1ccc(S(=O)(=O)NN)cc1</smiles><smiles>Cc1ccc(S(=O)(=O)N2CCCCC2)cc1</smiles>

\begin{tabular}{clclc}
\hline Entry & \multicolumn{1}{c}{ Catalyst } & $n /$ mmol & Solvent & Yield $\%$ \\
\hline 1 & $\mathrm{CuBr}_{2}$ & 2.5 & $\mathrm{CH}_{3} \mathrm{CN}$ & 66 \\
2 & $\mathrm{CuCl}_{2} \cdot 2 \mathrm{H}_{2} \mathrm{O}$ & 2.5 & $\mathrm{CH}_{3} \mathrm{CN}$ & 61 \\
3 & $\mathrm{Cu}(\mathrm{acac})_{2}$ & 2.5 & $\mathrm{CH}_{3} \mathrm{CN}$ & Trace \\
4 & $\mathrm{CuBr}$ & 2.5 & $\mathrm{CH}_{3} \mathrm{CN}$ & 39 \\
5 & $\mathrm{CuI}$ & 2.5 & $\mathrm{CH}_{3} \mathrm{CN}$ & 49 \\
6 & $\mathrm{FeCl}_{3}$ & 2.5 & $\mathrm{CH}_{3} \mathrm{CN}$ & 0 \\
7 & $\mathrm{NiCl}_{2} \bullet 6 \mathrm{H}_{2} \mathrm{O}$ & 2.5 & $\mathrm{CH}_{3} \mathrm{CN}$ & 0 \\
8 & $\mathrm{CuBr}_{2}$ & 2.5 & DMA & 73 \\
9 & $\mathrm{CuBr}_{2}$ & 2.5 & THF & 41 \\
10 & $\mathrm{CuBr}_{2}$ & 2.5 & EtOH & 36 \\
11 & $\mathrm{CuBr}_{2}$ & 2.5 & DMSO & 42 \\
12 & $\mathrm{CuBr}_{2}$ & 2.5 & Tol & 33 \\
13 & $\mathrm{CuBr}_{2}$ & 2.0 & DMA & 73 \\
14 & $\mathrm{CuBr}_{2}$ & 1.5 & DMA & 75 \\
$15^{b}$ & $\mathrm{CuBr}_{2}$ & 1.5 & DMA & 84 \\
$16^{b, c}$ & $\mathrm{CuBr}_{2}$ & 1.5 & DMA & 79 \\
$17^{b, d}$ & $\mathrm{CuBr}_{2}$ & 1.5 & DMA & 75 \\
\hline
\end{tabular}

${ }^{a}$ The reactions were carried out with 1 a $(0.5 \mathrm{mmol}), \mathbf{2 a}(\mathrm{n} \mathrm{mmol})$, catalyst $(0.025 \mathrm{mmol})$, solvent $(0.5 \mathrm{~mL})$ at $70{ }^{\circ} \mathrm{C}$ for $10 \mathrm{~h}$ under $\mathrm{O}_{2}$ balloon, isolated yield. ${ }^{b} 0.1 \mathrm{~mL}$ of DMA was used. ${ }^{c}$ The reaction was carried out at $80{ }^{\circ} \mathrm{C}$.

${ }^{d}$ The reaction was performed under air.

when prolinol was employed as the substrate, the sulfonylation reaction underwent smoothly to give the corresponding sulfonamide $\mathbf{3 a h}$ in a highly specific manner. Moreover, noncyclic amines were also well-tolerated. When secondary aliphatic amines were employed as the substrates, the corresponding products $\mathbf{3 a i} \sim \mathbf{3 a k}$ were obtained in $67 \% \sim 72 \%$ yields. It is noteworthy that the aromatic amine with much lower nucleophilicity was also a good substrate for this transformation, giving sulfonamide 3al in 64\% yield. When primary amines were employed as the substrates, the corresponding sulfonamides 3am $\sim$ 3an were obtained with lower yields.

To investigate the synthetic applicability of this copper-catalyzed aerobic sulfonamide synthesis, we tested the scale-up ability by using the reaction of $\mathbf{1 a}$ and $\mathbf{2 a}$. As shown in Scheme 2, the reaction can be easily scaled up to a gram scale $(10 \mathrm{mmol})$ with similar efficiency.

To gain more insights into this reaction, the radical trapping reagent (TEMPO or 1,1-diphenylethylene) was added into the reaction of $\mathbf{1 a}$ and $\mathbf{2 a}$. It was shown that the formation of sulfonamide 3aa was completely prohibited, suggesting a radical process maybe involved (Scheme 3 ).

Based on the control experiments and previous studies, ${ }^{[19]}$ a plausible mechanism for the formation of sulfon-
Table 2 Substrate scope of sulfonyl hydrazides ${ }^{a}$

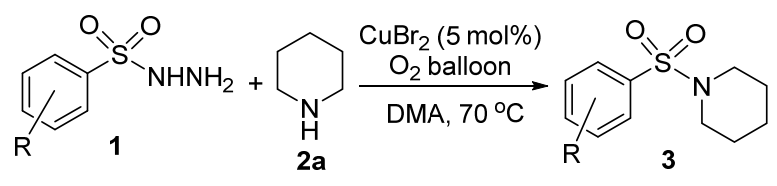<smiles>Cc1ccc(S(=O)(=O)N2CCCCC2)cc1</smiles>

3aa, $84 \%$<smiles>O=S(=O)(c1ccc(F)cc1)N1CCCCC1</smiles>

3da, $65 \%$<smiles>O=[N+]([O-])c1ccc(S(=O)(=O)N2CCCCC2)cc1</smiles>

3ga, $46 \%$<smiles>Cc1cc(C)c(S(=O)(=O)N2CCCCC2)c(C)c1</smiles>

3ja, $75 \%$<smiles>O=S(=O)(c1ccccc1)N1CCCCC1</smiles>

3ba, $84 \%$<smiles>COc1ccc(S(=O)(=O)N2CCCCC2)cc1</smiles>

3ca, $61 \%$<smiles>O=S(=O)(c1ccc(Cl)cc1)N1CCCCC1</smiles>

3ea, $77 \%$<smiles>Cc1ccccc1S(=O)(=O)N1CCCCC1</smiles><smiles>O=S(=O)(c1ccc2ccccc2c1)N1CCCCC1</smiles>

3ka, $78 \%$<smiles>O=S(=O)(c1ccc(Br)cc1)N1CCCCC1</smiles>

3fa, $74 \%$<smiles>Cc1cccc(S(=O)(=O)N2CCCCC2)c1</smiles>

3ia, $82 \%$

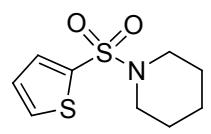

3la, $59 \%$
${ }^{a}$ The reactions were carried out with $1(0.5 \mathrm{mmol}), \mathbf{2 a}(1.5 \mathrm{mmol}), \mathrm{CuBr}_{2}$ $(0.025 \mathrm{mmol}), \mathrm{DMA}(0.1 \mathrm{~mL})$ at $70{ }^{\circ} \mathrm{C}$ for $10 \mathrm{~h}$ under $\mathrm{O}_{2}$ balloon.

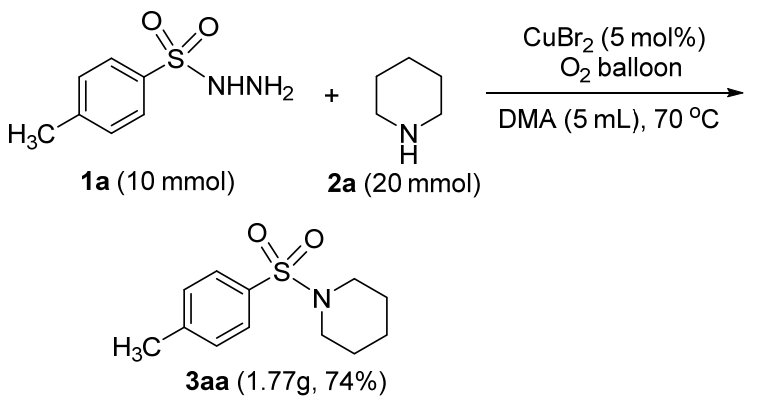

Scheme 2 Gram-scale synthesis

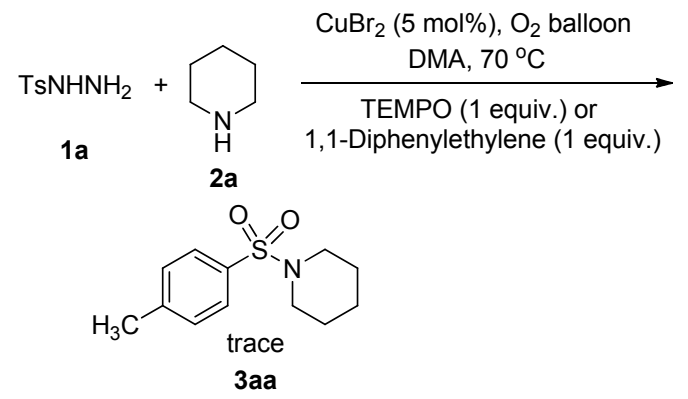

Scheme 3 Radical trapping experiments

amide 3 was proposed (Scheme 4). Aryl sulfonyl hydrazine 1 first coordinates with $\mathrm{CuBr}_{2}$ to form copper complex 4, which then undergoes an elimination step to afford intermediate 5. ${ }^{[18,20]}$ Subsequently, intermediate 5 undergoes a 
Table 3 Substrate Scope of Amines ${ }^{a}$

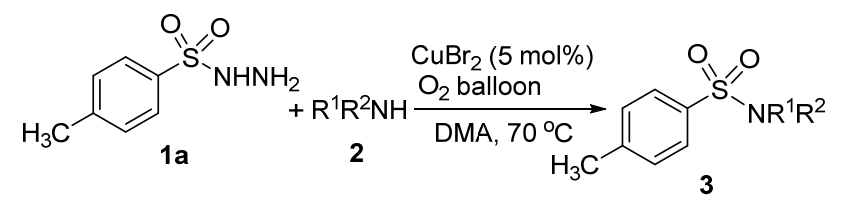

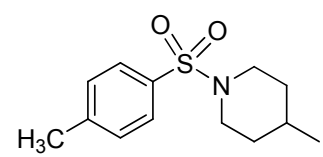

$3 a b, 84 \%$<smiles>Cc1ccc(S(=O)(=O)N2CCCCC2C)cc1</smiles>

3ad, $59 \%$<smiles>Cc1ccc(S(=O)(=O)N2CCOCC2)cc1</smiles>

3af, $83 \%$<smiles>Cc1ccc(S(=O)(=O)N2CCCC2CO)cc1</smiles>

3ah, $62 \%$<smiles>Cc1ccc(S(=O)(=O)N(Cc2ccccc2)Cc2ccccc2)cc1</smiles>

3aj, $71 \%$<smiles>Cc1ccc(S(=O)(=O)N(C)c2ccccc2)cc1</smiles>

3al, $64 \%$

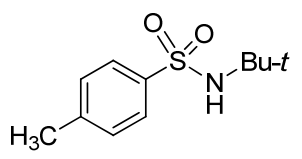

3an, 53\%<smiles>Cc1ccc(S(=O)(=O)N2CCCC(C)C2)cc1</smiles>

3ac, $83 \%$<smiles>Cc1ccc(S(=O)(=O)N2CCC(O)CC2)cc1</smiles>

3ae, $76 \%$<smiles>Cc1ccc(S(=O)(=O)N2CCCC2)cc1</smiles><smiles>Cc1ccc(S(=O)(=O)N(Br)[Ga]N(Br)Br)cc1</smiles>

3ai, $67 \%$<smiles>C=CCN(CC=C)S(=O)(=O)c1ccc(C)cc1</smiles><smiles>Cc1ccc(S(=O)(=O)NC(=O)c2ccccc2)cc1</smiles>

3am, 61\%
${ }^{a}$ The reactions were carried out with $\mathbf{1 a}(0.5 \mathrm{mmol}), \mathbf{2}(1.5 \mathrm{mmol}), \mathrm{CuBr}_{2}$ $(0.025 \mathrm{mmol})$, DMA $(0.1 \mathrm{~mL})$ at $70{ }^{\circ} \mathrm{C}$ for $10 \mathrm{~h}$ under $\mathrm{O}_{2}$ balloon.

single electron oxidation to produce the sulfonyl radical 6 with the extrusion of molecular nitrogen.$^{[11,16 a]}$ Meanwhile, coordination of $\mathrm{Cu}$ (II) to the amine 2 gives intermediate $\mathbf{7}$, which could be trapped by sulfonyl radical $\mathbf{6}$ to deliver the desired product 3. Meanwhile, one molecule of $\mathrm{Cu}(\mathrm{I})$ is generated, which could be reoxidized to $\mathrm{Cu}(\mathrm{II})$ by aerobic oxidation. ${ }^{[21]}$

\section{Conclusions}

We have developed a copper-catalyzed aerobic denitrogenative amination protocol and demonstrated its utility over a wide range of sulfonyl hydrazides and amines. In

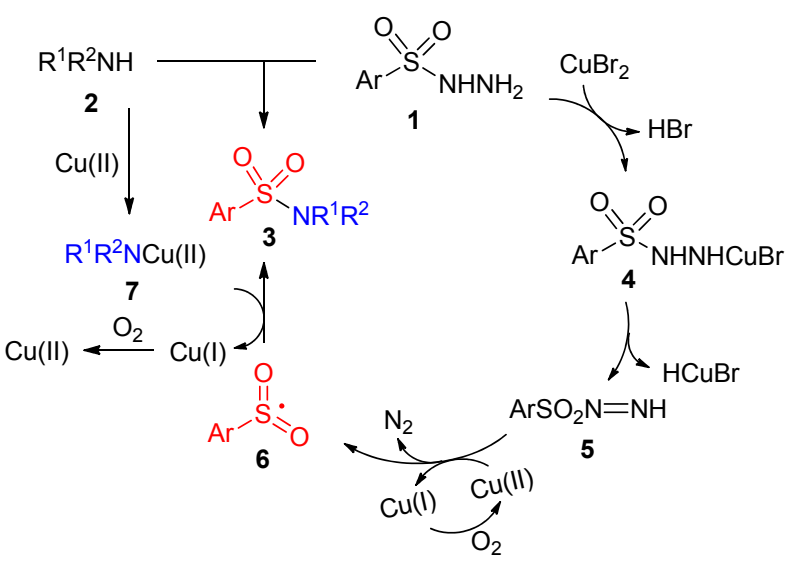

Scheme 4 Proposed mechanism for the formation of sulfonamide

contrast to previously described methods, this reaction employs copper $/ \mathrm{O}_{2}$ as the catalytic system, utilizes easily available starting materials and generates $\mathrm{N}_{2}$ as the only byproduct, which provides an environmentally benign and low-cost synthetic route for sulfonamides. In addition, the operational simplicity and mild reaction conditions allow for the synthesis of a diverse collection of valuable sulfonamides products with moderate to excellent yields.

\section{Experimental section}

\subsection{Instruments and reagents}

NMR spectra were recorded on 300 or $400 \mathrm{MHz}$ NMR spectrometer with $\mathrm{CDCl}_{3}$ as the solvent and tetramethylsilane (TMS) as the internal standard. Chemical shifts were reported downfield from TMS at 0.00 and referenced to the $\mathrm{CDCl}_{3}$ at $\delta 7.26$ (for ${ }^{1} \mathrm{H} \mathrm{NMR}$ ) or 77.16 (for ${ }^{13} \mathrm{C}$ NMR). Melting points were determined on a melting point apparatus and are uncorrected. The amines were commercially available and were used without further purification. DMA was distilled over calcium hydride under reduced pressure.

\subsection{Experimental method}

To a solution of sulfonyl hydrazine 1a (93 $\mathrm{mg}, 0.5$ mmol) and piperdine $2 \mathbf{a}(128 \mathrm{mg}, 1.5 \mathrm{mmol})$ in distilled DMA $(0.1 \mathrm{~mL})$ was added $\mathrm{CuBr}_{2}(5 \mathrm{mg}, 0.025 \mathrm{mmol})$ at room temperature. Then, the reaction mixture was stirred at $70{ }^{\circ} \mathrm{C}$ for $10 \mathrm{~h}$ under an oxygen balloon. After the reaction was completed, the resulting mixture was extracted with EtOAc $(10 \mathrm{~mL} \times 3)$, washed with aqueous $\mathrm{NaCl}$, dried with $\mathrm{Na}_{2} \mathrm{SO}_{4}$. Then the solvent was removed under reduced pressure, and the resulting mixture was purified by silica gel column chromatography (Hexane/EtOAc, $V: V=4: 1 \sim 1: 1)$ to afford the desired product 3aa (100 mg, 84\%) as a white solid.

1-Tosylpiperidine (3aa): ${ }^{[16 a]}$ Isolated yield $84 \%$ (100 mg). White solid, m.p. $118 \sim 120{ }^{\circ} \mathrm{C} ;{ }^{1} \mathrm{H}$ NMR $(400 \mathrm{MHz}$, $\left.\mathrm{CDCl}_{3}\right) \delta: 1.40 \sim 1.44(\mathrm{~m}, 2 \mathrm{H}), 1.61 \sim 1.66(\mathrm{~m}, 4 \mathrm{H}), 2.43$ (s, 3H), 2.97 (t, $J=5.6 \mathrm{~Hz}, 4 \mathrm{H}), 7.31(\mathrm{~d}, J=8.0 \mathrm{~Hz}, 2 \mathrm{H})$, $7.63 \sim 7.65(\mathrm{~m}, 2 \mathrm{H}) ;{ }^{13} \mathrm{C} \mathrm{NMR}\left(100 \mathrm{MHz}, \mathrm{CDCl}_{3}\right) \delta: 21.6$, 
23.7, 25.3, 47.1, 127.9, 129.7, 133.5, 143.4; MS (EI) $m / z$ : $239\left(\mathrm{M}^{+}\right)$.

1-(Phenylsulfonyl)piperidine (3ba): ${ }^{[15]}$ Isolated yield $84 \%$ (95 mg). White solid, m.p. $116 \sim 119{ }^{\circ} \mathrm{C}$; ${ }^{1} \mathrm{H}$ NMR $\left(400 \mathrm{MHz}, \mathrm{CDCl}_{3}\right) \delta: 1.40(\mathrm{~m}, 2 \mathrm{H}), 1.75 \sim 1.56(\mathrm{~m}, 4 \mathrm{H})$, $2.98(\mathrm{t}, J=4.0 \mathrm{~Hz}, 4 \mathrm{H}), 7.55 \sim 7.47(\mathrm{~m}, 2 \mathrm{H}), 7.62 \sim 7.55$ $(\mathrm{m}, 1 \mathrm{H}), 7.80 \sim 7.70(\mathrm{~m}, 2 \mathrm{H}) ;{ }^{13} \mathrm{C} \mathrm{NMR}(100 \mathrm{MHz}$, $\left.\mathrm{CDCl}_{3}\right) \delta: 23.6,25.2,47.0,127.7,129.0,132.7,136.3$; IR (KBr) $v$ : 694, 822, 1500, 1601, $2915 \mathrm{~cm}^{-1}$; MS (EI) $\mathrm{m} / z$ : $225\left(\mathrm{M}^{+}\right)$.

1-((4-Methoxyphenyl)sulfonyl)piperidine (3ca): ${ }^{[17 \mathrm{c}]}$ Isolated yield $61 \%$ (78 mg). White solid, m.p. $111 \sim 112{ }^{\circ} \mathrm{C}$; ${ }^{1} \mathrm{H}$ NMR $\left(400 \mathrm{MHz}, \mathrm{CDCl}_{3}\right) \delta: 1.41 \sim 1.43(\mathrm{~m}, 2 \mathrm{H})$, $1.61 \sim 1.65(\mathrm{~m}, 4 \mathrm{H}), 2.96(\mathrm{t}, J=5.6 \mathrm{~Hz}, 4 \mathrm{H}), 3.87(\mathrm{~s}, 3 \mathrm{H})$, $6.97 \sim 7.00(\mathrm{~m}, 2 \mathrm{H}), 7.68 \sim 7.70(\mathrm{~m}, 2 \mathrm{H}) ;{ }^{13} \mathrm{C}$ NMR $(100$ $\left.\mathrm{MHz}, \mathrm{CDCl}_{3}\right) \delta: 23.7,25.3,47.1,55.7,114.2,128.2,129.9$, 163.0; IR (KBr) v: 698, 822, 1602, $2924 \mathrm{~cm}^{-1}$; MS (EI) $m / z: 255\left(\mathrm{M}^{+}\right)$.

1-((4-Fluorophenyl)sulfonyl)piperidine (3da): ${ }^{[17 d]}$ Isolated yield $65 \%(79 \mathrm{mg})$. White solid, m.p. $131 \sim 132{ }^{\circ} \mathrm{C}$; ${ }^{1} \mathrm{H}$ NMR $\left(300 \mathrm{MHz}, \mathrm{CDCl}_{3}\right) \delta: 1.39 \sim 1.47(\mathrm{~m}, 2 \mathrm{H})$, $1.61 \sim 1.69(\mathrm{~m}, 4 \mathrm{H}), 2.99(\mathrm{t}, J=7.2 \mathrm{~Hz}, 4 \mathrm{H}), 7.18 \sim 7.23$ $(\mathrm{m}, 2 \mathrm{H}), 7.75 \sim 7.80(\mathrm{~m}, 2 \mathrm{H}) ;{ }^{13} \mathrm{C} \mathrm{NMR}\left(75 \mathrm{MHz}, \mathrm{CDCl}_{3}\right)$ $\delta: 23.6,25.3,47.0,116.3(\mathrm{~d}, J=22.5 \mathrm{~Hz}), 130.4(\mathrm{~d}, J=9$ $\mathrm{Hz}), 132.7$ (d, $J=3 \mathrm{~Hz}), 165.2$ (d, $J=252.8 \mathrm{~Hz}$ ); IR (KBr) $v: 696,821,1249,1361,1520,1602,2917 \mathrm{~cm}^{-1}$; MS (EI) $m / z: 243\left(\mathrm{M}^{+}\right)$.

1-((4-Chlorophenyl)sulfonyl)piperidine (3ea): ${ }^{[11]}$ Isolated yield 77\% (100 mg). White solid, m.p. 112 $113{ }^{\circ} \mathrm{C}$; ${ }^{1} \mathrm{H}$ NMR $\left(400 \mathrm{MHz}, \mathrm{CDCl}_{3}\right) \delta: 1.41 \sim 1.47(\mathrm{~m}, 2 \mathrm{H})$, $1.62 \sim 1.68(\mathrm{~m}, 4 \mathrm{H}), 2.99(\mathrm{t}, J=5.6 \mathrm{~Hz}, 4 \mathrm{H}), 7.48 \sim 7.52$ $(\mathrm{m}, 2 \mathrm{H}), 7.68 \sim 7.71(\mathrm{~m}, 2 \mathrm{H}) ;{ }^{13} \mathrm{C}$ NMR $(100 \mathrm{MHz}$, $\left.\mathrm{CDCl}_{3}\right) \delta: 23.6,25.3,47.0,129.2,129.4,135.2,139.2$; IR (KBr) $v:$ 694, 841, 1246, 1502, $2924 \mathrm{~cm}^{-1}$; MS (EI) $\mathrm{m} / z$ : $260\left(\mathrm{M}^{+}\right)$.

1-((4-Bromophenyl)sulfonyl)piperidine (3fa): $:^{[17 \mathrm{e}]}$ Isolated yield $74 \%$ (113 mg). White solid, m.p. $123 \sim 124{ }^{\circ} \mathrm{C}$; ${ }^{1} \mathrm{H}$ NMR $\left(400 \mathrm{MHz}, \mathrm{CDCl}_{3}\right) \delta: 1.33 \sim 1.39(\mathrm{~m}, 2 \mathrm{H})$, $1.54 \sim 1.60(\mathrm{~m}, 4 \mathrm{H}), 2.91(\mathrm{t}, J=5.6 \mathrm{~Hz}, 4 \mathrm{H}), 7.53 \sim 7.56$ $(\mathrm{m}, 2 \mathrm{H}), 7.58 \sim 7.61(\mathrm{~m}, 2 \mathrm{H}) ;{ }^{13} \mathrm{C}$ NMR $(100 \mathrm{MHz}$, $\left.\mathrm{CDCl}_{3}\right) \delta: 23.6,25.3,47.0,127.7,129.3,132.4,135.7 . \mathrm{IR}$ (KBr) $v: 696,824,1602,2929 \mathrm{~cm}^{-1}$; MS (EI) $\mathrm{m} / z: 304$ $\left(\mathrm{M}^{+}\right)$.

1-((4-Nitrophenyl)sulfonyl)piperidine (3ga): Isolated yield $46 \%$ (62 mg). White solid, m.p. $179 \sim 180{ }^{\circ} \mathrm{C} ;{ }^{1} \mathrm{H}$ NMR $\left(400 \mathrm{MHz}, \mathrm{CDCl}_{3}\right) \delta: 1.43 \sim 1.49(\mathrm{~m}, 2 \mathrm{H}), 1.62 \sim$ $1.69(\mathrm{~m}, 4 \mathrm{H}), 3.06(\mathrm{t}, J=5.6 \mathrm{~Hz}, 4 \mathrm{H}), 7.93 \sim 7.96(\mathrm{~m}, 2 \mathrm{H})$, $8.36 \sim 8.40(\mathrm{~m}, 2 \mathrm{H}) ;{ }^{13} \mathrm{C}$ NMR $\left(100 \mathrm{MHz}, \mathrm{CDCl}_{3}\right) \delta: 23.5$, 25.3, 47.1, 124.4, 128.9, 142.9, 150.2; IR (KBr) v: 698, $704,829,1156,1204,1601,2933 \mathrm{~cm}^{-1}$. HRMS calcd $\mathrm{C}_{11} \mathrm{H}_{14} \mathrm{~N}_{2} \mathrm{O}_{4} \mathrm{~S} 270.0674$; found 270.0671

1-(o-Tolylsulfonyl)piperidine (3ha): ${ }^{[17 \mathrm{e}]}$ Isolated yield $81 \%$ (97 mg). White solid, m.p. $119 \sim 121{ }^{\circ} \mathrm{C} ;{ }^{1} \mathrm{H}$ NMR $\left(300 \mathrm{MHz}, \mathrm{CDCl}_{3}\right) \delta: 1.52 \sim 1.55(\mathrm{~m}, 2 \mathrm{H}), 1.59 \sim 1.66(\mathrm{~m}$, $4 \mathrm{H}), 2.64(\mathrm{~s}, 3 \mathrm{H}), 3.15(\mathrm{t}, J=5.4 \mathrm{~Hz}, 4 \mathrm{H}), 7.30 \sim 7.33(\mathrm{~m}$, 2H), $7.43 \sim 7.45(\mathrm{~m}, 1 \mathrm{H}), 7.90(\mathrm{~d}, J=9 \mathrm{~Hz}, 1 \mathrm{H}) ;{ }^{13} \mathrm{C} \mathrm{NMR}$ $\left(75 \mathrm{MHz}, \mathrm{CDCl}_{3}\right) \delta: 20.8,23.9,25.5,46.1,126.0,130.3$,
132.7, 132.8, 136.3, 138.1; IR (KBr) v: 698, 769, 1506, $2926 \mathrm{~cm}^{-1}$; MS (EI) $m / z: 239\left(\mathrm{M}^{+}\right)$.

1-(m-Tolylsulfonyl)piperidine (3ia): ${ }^{[17 \mathrm{e}]}$ Isolated yield $82 \%$ (98 mg). White solid, m.p. $136 \sim 137{ }^{\circ} \mathrm{C} ;{ }^{1} \mathrm{H}$ NMR $\left(300 \mathrm{MHz}, \mathrm{CDCl}_{3}\right) \delta: 1.38 \sim 1.46(\mathrm{~m}, 2 \mathrm{H}), 1.61 \sim 1.68(\mathrm{~m}$, $4 \mathrm{H}), 2.44(\mathrm{~s}, 3 \mathrm{H}), 2.99(\mathrm{t}, J=5.1 \mathrm{~Hz}, 4 \mathrm{H}), 7.37 \sim 7.44(\mathrm{~m}$, 2H), $7.54 \sim 7.56(\mathrm{~m}, 2 \mathrm{H}) ;{ }^{13} \mathrm{C}$ NMR $\left(75 \mathrm{MHz}, \mathrm{CDCl}_{3}\right) \delta$ : 21.5, 23.7, 25.3, 47.1, 125.0, 128.1, 128.9, 133.5, 136.4, 139.2. IR (KBr) $v:$ 691, 792, 1316, 1590, $2921 \mathrm{~cm}^{-1}$; MS (EI) $m / z: 239\left(\mathrm{M}^{+}\right)$.

1-(Mesitylsulfonyl)piperidine (3ja): Isolated yield 75\% (100 mg). White solid, m.p. 184 $185{ }^{\circ} \mathrm{C} ;{ }^{1} \mathrm{H}$ NMR $(400$ $\left.\mathrm{MHz}, \mathrm{CDCl}_{3}\right) \delta: 1.55 \sim 1.59(\mathrm{~m}, 6 \mathrm{H}), 2.30(\mathrm{~s}, 3 \mathrm{H}), 2.62(\mathrm{~s}$, $6 \mathrm{H}), 3.13(\mathrm{t}, J=5.2 \mathrm{~Hz}, 4 \mathrm{H}), 6.94(\mathrm{~s}, 2 \mathrm{H}) ;{ }^{13} \mathrm{C} \mathrm{NMR}(100$ $\left.\mathrm{MHz}, \mathrm{CDCl}_{3}\right) \delta: 21.0,22.8,24.1,25.3,45.0,131.9,132.1$, 140.5, 142.4; IR (KBr) v: 697, 853, 1020, 1516, 2927 $\mathrm{cm}^{-1}$; MS (EI) $m / z: 267\left(\mathrm{M}^{+}\right)$.

1-(Naphthalen-2-ylsulfonyl)piperidine (3ka): Isolated yield $78 \%$ (107 mg). White solid, m.p. 133 134 ${ }^{\circ} \mathrm{C} ;{ }^{1} \mathrm{H}$ NMR $\left(300 \mathrm{MHz}, \mathrm{CDCl}_{3}\right) \delta: 1.36 \sim 1.44(\mathrm{~m}, 2 \mathrm{H}), 1.61 \sim$ $1.69(\mathrm{~m}, 4 \mathrm{H}), 3.06(\mathrm{t}, J=5.4 \mathrm{~Hz}, 4 \mathrm{H}), 7.59 \sim 7.68(\mathrm{~m}, 2 \mathrm{H})$, $7.74 \sim 7.77(\mathrm{~m}, 1 \mathrm{H}), 7.91 \sim 7.97(\mathrm{~m}, 3 \mathrm{H}), 8.32(\mathrm{~s}, 1 \mathrm{H}) ;{ }^{13} \mathrm{C}$ NMR $\left(75 \mathrm{MHz}, \mathrm{CDCl}_{3}\right) \delta: 23.6,25.3,47.1,123.2,127.6$, $128.0,128.8,129.0,129.2,129.3,132.4,133.8,134.9$; IR (KBr) $v:$ 698, 859, 1517, $2929 \mathrm{~cm}^{-1}$. HRMS calcd $\mathrm{C}_{15} \mathrm{H}_{17} \mathrm{NO}_{2} \mathrm{~S} 275.0980$; found 275.0977 .

1-(Thiophen-2-ylsulfonyl)piperidine (3la): Isolated yield $59 \%$ (68 mg). White solid, m.p. $160 \sim 161{ }^{\circ} \mathrm{C} ;{ }^{1} \mathrm{H}$ NMR $\left(400 \mathrm{MHz}, \mathrm{CDCl}_{3}\right) \delta: 1.42 \sim 1.48(\mathrm{~m}, 2 \mathrm{H}), 1.65 \sim 1.71(\mathrm{~m}$, $4 \mathrm{H}), 3.04(\mathrm{t}, J=5.6 \mathrm{~Hz}, 4 \mathrm{H}), 7.13 \sim 7.15(\mathrm{~m}, 1 \mathrm{H}), 7.51 \sim$ $7.52(\mathrm{~m}, 1 \mathrm{H}), 7.59 \sim 7.61(\mathrm{~m}, 1 \mathrm{H}) ;{ }^{13} \mathrm{C} \mathrm{NMR}(100 \mathrm{MHz}$, $\left.\mathrm{CDCl}_{3}\right) \delta: 23.6,25.2,47.1,127.6,131.8,132.2,137.1 . \mathrm{IR}$ (KBr) v: 698, 1067, 1127, $2927 \mathrm{~cm}^{-1}$. HRMS calcd $\mathrm{C}_{9} \mathrm{H}_{13} \mathrm{NO}_{2} \mathrm{~S}_{2} 231.0388$; found 231.0385.

4-Methyl-1-tosylpiperidine (3ab): Isolated yield $84 \%$ (106 mg). White solid, m.p. 165 166 ${ }^{\circ} \mathrm{C} ;{ }^{1} \mathrm{H}$ NMR $(400$ $\left.\mathrm{MHz}, \mathrm{CDCl}_{3}\right) \delta: 0.90(\mathrm{~d}, J=5.6 \mathrm{~Hz}, 3 \mathrm{H}), 1.26 \sim 1.31(\mathrm{~m}$, $3 \mathrm{H}), 1.61 \sim 1.67(\mathrm{~m}, 2 \mathrm{H}), 2.20-2.26(\mathrm{~m}, 2 \mathrm{H}), 2.43(\mathrm{~s}, 3 \mathrm{H})$, $3.71-3.75(\mathrm{~m}, 2 \mathrm{H}), 7.31(\mathrm{~d}, J=8.0 \mathrm{~Hz}, 2 \mathrm{H}), 7.62 \sim 7.65$ $(\mathrm{m}, 2 \mathrm{H}) ;{ }^{13} \mathrm{C} \mathrm{NMR}\left(100 \mathrm{MHz}, \mathrm{CDCl}_{3}\right) \delta: 21.2,21.6,30.3$, $33.5,46.5,127.9,129.7,133.5,143.4$. IR (KBr) v: 696, 1091, 1519, $2926 \mathrm{~cm}^{-1}$. HRMS calcd $\mathrm{C}_{13} \mathrm{H}_{19} \mathrm{NO}_{2} \mathrm{~S}$ 253.1136; found 253.1131 .

3-Methyl-1-tosylpiperidine (3ac): Isolated yield 83\% (105 mg). White solid, m.p. $134 \sim 135{ }^{\circ} \mathrm{C} ;{ }^{1} \mathrm{H}$ NMR $(400$ $\left.\mathrm{MHz}, \mathrm{CDCl}_{3}\right) \delta: 0.76 \sim 0.86(\mathrm{~m}, 1 \mathrm{H}), 0.88(\mathrm{~d}, J=5.6 \mathrm{~Hz}, 3$ $\mathrm{H}), 1.60 \sim 1.76(\mathrm{~m}, 4 \mathrm{H}), 1.86 \sim 1.91(\mathrm{~m}, 1 \mathrm{H}), 2.18 \sim 2.24$ $(\mathrm{m}, 1 \mathrm{H}), 2.43(\mathrm{~s}, 3 \mathrm{H}), 3.59 \sim 3.66(\mathrm{~m}, 2 \mathrm{H}), 7.31(\mathrm{~d}, J=8.0$ $\mathrm{Hz}, 2 \mathrm{H}), 7.62 \sim 7.65(\mathrm{~m}, 2 \mathrm{H}) ;{ }^{13} \mathrm{C} \mathrm{NMR}(100 \mathrm{MHz}$, $\left.\mathrm{CDCl}_{3}\right) \delta: 19.1,21.6,24.8,30.8,32.2,46.6,53.4,127.8$, 129.7, 133.6, 143.4; IR (KBr) v: 696, 843, 1519, 2923 $\mathrm{cm}^{-1}$. HRMS calcd $\mathrm{C}_{13} \mathrm{H}_{19} \mathrm{NO}_{2} \mathrm{~S}$ 253.1136; found 253.1133 .

2-Methyl-1-tosylpiperidine (3ad): Isolated yield 59\% (75 mg). White solid, m.p. $115 \sim 117{ }^{\circ} \mathrm{C} ;{ }^{1} \mathrm{H}$ NMR (400 $\left.\mathrm{MHz}, \mathrm{CDCl}_{3}\right) \delta: 1.08(\mathrm{~d}, J=5.6 \mathrm{~Hz}, 3 \mathrm{H}), 1.42 \sim 1.50(\mathrm{~m}$, $2 \mathrm{H}), 1.52 \sim 1.61(\mathrm{~m}, 4 \mathrm{H}), 2.41(\mathrm{~s}, 3 \mathrm{H}), 2.94 \sim 3.01(\mathrm{~m}$, 
$1 \mathrm{H}), 3.67 \sim 3.72(\mathrm{~m}, 1 \mathrm{H}), 4.21 \sim 4.25(\mathrm{~m}, 1 \mathrm{H}), 7.26 \sim 7.28$ $(\mathrm{m}, 2 \mathrm{H}), 7.69 \sim 7.72(\mathrm{~m}, 2 \mathrm{H}) ;{ }^{13} \mathrm{C} \mathrm{NMR}(100 \mathrm{MHz}$, $\left.\mathrm{CDCl}_{3}\right) \delta: 15.5,18.3,21.6,25.4,30.5,40.4,48.6,127.1$, 129.7, 138.6, 142. 9; IR (KBr) v: 696, 822, 1249, 1360, 1521, 1603, $2918 \mathrm{~cm}^{-1}$. HRMS calcd $\mathrm{C}_{13} \mathrm{H}_{19} \mathrm{NO}_{2} \mathrm{~S}$ 253.1136; found 253.1130.

1-Tosylpiperidin-4-ol (3ae): Isolated yield 76\% (97 mg). White solid, m.p. $107 \sim 109{ }^{\circ} \mathrm{C} ;{ }^{1} \mathrm{H}$ NMR $(300 \mathrm{MHz}$, $\left.\mathrm{CDCl}_{3}\right) \delta: 1.61 \sim 1.70(\mathrm{~m}, 2 \mathrm{H}), 1.89 \sim 1.92(\mathrm{~m}, 2 \mathrm{H}), 2.43$ $(\mathrm{s}, 3 \mathrm{H}), 2.81 \sim 2.89(\mathrm{~m}, 2 \mathrm{H}), 2.99(\mathrm{~s}, 1 \mathrm{H}), 3.28 \sim 3.35(\mathrm{~m}$, 2H), $3.73 \sim 3.77(\mathrm{~m}, 1 \mathrm{H}), 7.32(\mathrm{~d}, J=10.4 \mathrm{~Hz}, 2 \mathrm{H}), 7.64$ $(\mathrm{d}, J=10.8 \mathrm{~Hz}, 2 \mathrm{H}) ;{ }^{13} \mathrm{C} \mathrm{NMR}\left(100 \mathrm{MHz}, \mathrm{CDCl}_{3}\right) \delta: 21.6$, $33.3,43.3,65.8,127.7,129.7,133.2,143.6$. IR (KBr) $v$ : $696,821,1250,1363,1520,2916 \mathrm{~cm}^{-1}$. HRMS calcd $\mathrm{C}_{12} \mathrm{H}_{17} \mathrm{NO}_{3} \mathrm{~S}$ 255.0929; found 255.0923.

4-Tosylmorpholine (3af): ${ }^{[14]}$ Isolated yield 83\% (101 mg). White solid, m.p. $137 \sim 138{ }^{\circ} \mathrm{C} ;{ }^{1} \mathrm{H}$ NMR $(300 \mathrm{MHz}$, $\left.\mathrm{CDCl}_{3}\right) \delta: 2.45(\mathrm{~s}, 3 \mathrm{H}), 2.99(\mathrm{t}, J=4.8 \mathrm{~Hz}, 4 \mathrm{H}), 3.74(\mathrm{t}, J=$ $4.8 \mathrm{~Hz}, 4 \mathrm{H}), 7.34(\mathrm{~d}, J=8.1 \mathrm{~Hz}, 2 \mathrm{H}), 7.64(\mathrm{~d}, J=8.1 \mathrm{~Hz}$, $2 \mathrm{H}) ;{ }^{13} \mathrm{C} \mathrm{NMR}\left(75 \mathrm{MHz}, \mathrm{CDCl}_{3}\right) \delta: 21.6,46.1,66.2,128.0$, 129.9, 132.3, 144.1; MS (EI) $m / z: 241\left(\mathrm{M}^{+}\right)$.

1-Tosylpyrrolidine (3ag): ${ }^{[13]}$ Isolated yield 74\% (83 mg). White solid, m.p. $161 \sim 162{ }^{\circ} \mathrm{C} ;{ }^{1} \mathrm{H}$ NMR $(400 \mathrm{MHz}$, $\left.\mathrm{CDCl}_{3}\right) \delta: 1.73 \sim 1.76(\mathrm{~m}, 4 \mathrm{H}), 2.43(\mathrm{~s}, 3 \mathrm{H}), 3.21 \sim 3.25$ $(\mathrm{m}, 4 \mathrm{H}), 7.31 \sim 7.33(\mathrm{~m}, 2 \mathrm{H}), 7.70 \sim 7.73(\mathrm{~m}, 2 \mathrm{H}) ;{ }^{13} \mathrm{C}$ NMR (100 MHz, $\left.\mathrm{CDCl}_{3}\right) \delta: 21.6,25.3,48.0,127.7,129.7$, 134.1, 143.4; $\mathrm{MS}(\mathrm{EI}) \mathrm{m} / \mathrm{z}: 225\left(\mathrm{M}^{+}\right)$.

(1-Tosylpyrrolidin-2-yl)methanol (3ah): Isolated yield $62 \%$ (79 mg). White solid, m.p. 169 170 ${ }^{\circ} \mathrm{C} ;{ }^{1} \mathrm{H}$ NMR $\left(400 \mathrm{MHz}, \mathrm{CDCl}_{3}\right) \delta: 1.42 \sim 1.48(\mathrm{~m}, 1 \mathrm{H}), 1.66 \sim 1.83(\mathrm{~m}$, $4 \mathrm{H}), 2.44(\mathrm{~s}, 3 \mathrm{H}), 3.23 \sim 3.29(\mathrm{~m}, 1 \mathrm{H}), 3.62 \sim 3.69(\mathrm{~m}$, $3 \mathrm{H}), 7.28 \sim 7.35(\mathrm{~m}, 2 \mathrm{H}), 7.72 \sim 7.75(\mathrm{~m}, 2 \mathrm{H}) ;{ }^{13} \mathrm{C} \mathrm{NMR}$ $\left(100 \mathrm{MHz}, \mathrm{CDCl}_{3}\right) \delta: 21.6,24.3,29.0,29.8,50.1,61.9$, 65.9, 127.7, 129.9, 134.0, 143.9; IR (KBr) v: 694, 1089, $1518,1315,1598,2921 \mathrm{~cm}^{-1}$. HRMS calcd $\mathrm{C}_{12} \mathrm{H}_{17} \mathrm{NO}_{3} \mathrm{~S}$ 255.0929; found 255.0921.

$N, N$-Dibutyl-4-methylbenzenesulfonamide (3ai): ${ }^{[22]}$ Isolated yield $67 \%(95 \mathrm{mg})$. White solid, m.p. 167 168 ${ }^{\circ} \mathrm{C}$; ${ }^{1} \mathrm{H}$ NMR $\left(300 \mathrm{MHz}, \mathrm{CDCl}_{3}\right) \delta: 0.83(\mathrm{t}, J=7.5 \mathrm{~Hz}, 6 \mathrm{H})$, $1.17 \sim 1.29(\mathrm{~m}, 4 \mathrm{H}), 1.39 \sim 1.51(\mathrm{~m}, 4 \mathrm{H}), 2.36(\mathrm{~s}, 3 \mathrm{H})$, $3.03(\mathrm{t}, J=7.5 \mathrm{~Hz}, 4 \mathrm{H}), 7.22(\mathrm{~d}, J=8.4 \mathrm{~Hz}, 2 \mathrm{H}), 7.62$ (d, $J=8.4 \mathrm{~Hz}, 2 \mathrm{H}) ;{ }^{13} \mathrm{C} \mathrm{NMR}\left(75 \mathrm{MHz}, \mathrm{CDCl}_{3}\right) \delta: 13.8,20.1$, 21.6, 30.9, 48.1, 127.2, 129.6, 137.3, 143.0; IR (KBr) $v$ : 698, 1065, 1126, $2925 \mathrm{~cm}^{-1}$; MS (EI) $\mathrm{m} / \mathrm{z}: 283\left(\mathrm{M}^{+}\right)$.

$N, N$-Dibenzyl-4-methylbenzenesulfonamide (3aj): $:^{[16 a]}$ Isolated yield $71 \%(125 \mathrm{mg})$. White solid, m.p. 138 $139{ }^{\circ} \mathrm{C} ;{ }^{1} \mathrm{H}$ NMR (400 MHz, $\left.\mathrm{CDCl}_{3}\right) \delta: 2.40$ (s, 3H), 4.09 $(\mathrm{s}, 4 \mathrm{H}), 7.17 \sim 7.26(\mathrm{~m}, 4 \mathrm{H}), 7.26 \sim 7.34(\mathrm{~m}, 8 \mathrm{H}), 7.63(\mathrm{~d}$, $J=8.4 \mathrm{~Hz}, 2 \mathrm{H}) ;{ }^{13} \mathrm{C} \mathrm{NMR}\left(100 \mathrm{MHz}, \mathrm{CDCl}_{3}\right) \delta: 21.6$, $50.7,127.4,127.7,128.5,128.7,129.8,135.8,137.9$, 143.4; MS (EI) $m / z: 351\left(\mathrm{M}^{+}\right)$.

$N, N$-Diallyl-4-methylbenzenesulfonamide $\quad(\mathbf{3 a k}):^{[16 \mathrm{a}]}$ Isolated yield $72 \%$ (90 mg). White foam; ${ }^{1} \mathrm{H}$ NMR (400 $\left.\mathrm{MHz}, \mathrm{CDCl}_{3}\right) \delta: 2.43(\mathrm{~s}, 3 \mathrm{H}), 3.80(\mathrm{~d}, J=6.3 \mathrm{~Hz}, 4 \mathrm{H})$, $5.24 \sim 5.04(\mathrm{~m}, 4 \mathrm{H}), 5.61(\mathrm{~m}, 2 \mathrm{H}), 7.29(\mathrm{t}, J=6.5 \mathrm{~Hz}, 2 \mathrm{H})$, $7.70(\mathrm{~d}, J=8.3 \mathrm{~Hz}, 2 \mathrm{H}) ;{ }^{13} \mathrm{C} \mathrm{NMR}\left(100 \mathrm{MHz}, \mathrm{CDCl}_{3}\right) \delta$ : 21.6, 49.4, 119.0, 127.2, 129.8, 132.7, 137.5, 143.3; MS
(EI) $m / z: 251\left(\mathrm{M}^{+}\right)$.

$N$,4-Dimethyl- $N$-phenylbenzenesulfonamide $\quad$ (3al): $:^{[22]}$ Isolated yield $64 \%$ (84 mg). White solid, m.p. 86 $\sim 88{ }^{\circ} \mathrm{C}$; ${ }^{1} \mathrm{H}$ NMR (400 MHz, $\mathrm{CDCl}_{3}$ ) $\delta: 2.41(\mathrm{~s}, 3 \mathrm{H}), 3.16(\mathrm{~s}, 3 \mathrm{H})$, $7.05 \sim 7.13(\mathrm{~m}, 2 \mathrm{H}), 7.20 \sim 7.33(\mathrm{~m}, 5 \mathrm{H}), 7.42(\mathrm{~d}, J=8.2$ $\mathrm{Hz}, 2 \mathrm{H}) ;{ }^{13} \mathrm{C}$ NMR $\left(100 \mathrm{MHz}, \mathrm{CDCl}_{3}\right) \delta: 21.6,38.2,126.7$, $127.3,128.0,128.9,129.4,133.6,141.7,143.6$; MS (EI) $m / z: 261\left(\mathrm{M}^{+}\right)$.

$N$-Butyl-4-methylbenzenesulfonamide (3am): ${ }^{[13]}$ Isolated yield $61 \%$ (69 mg). White solid, m.p. $123 \sim 124{ }^{\circ} \mathrm{C} ;{ }^{1} \mathrm{H}$ NMR (300 MHz, $\left.\mathrm{CDCl}_{3}\right) \delta: 0.85(\mathrm{t}, J=7.2 \mathrm{~Hz}, 3 \mathrm{H})$, $1.25 \sim 1.33(\mathrm{~m}, 2 \mathrm{H}), 1.39 \sim 1.47(\mathrm{~m}, 2 \mathrm{H}), 2.43(\mathrm{~s}, 3 \mathrm{H})$, 2.94 (q, $J=6.9 \mathrm{~Hz}, 2 \mathrm{H}), 7.31(\mathrm{~d}, J=8.1 \mathrm{~Hz}, 2 \mathrm{H}), 7.75$ (d, $J=8.1 \mathrm{~Hz}, 2 \mathrm{H}) ;{ }^{13} \mathrm{C} \mathrm{NMR}\left(75 \mathrm{MHz}, \mathrm{CDCl}_{3}\right) \delta: 13.6,19.8$, 21.6, 31.7, 43.0, 127.2, 129.8, 137.2, 143.4; MS (EI) $\mathrm{m} / \mathrm{z}$ : $227\left(\mathrm{M}^{+}\right)$.

$N$-(Tert-butyl)-4-methylbenzenesulfonamide (3an): $:^{[13]}$ Isolated yield 53\% (60 mg). White solid, m.p. 103 $105{ }^{\circ} \mathrm{C} ;{ }^{1} \mathrm{H}$ NMR $\left(300 \mathrm{MHz}, \mathrm{CDCl}_{3}\right) \delta: 1.23$ (s, 9H), 2.42 $(\mathrm{s}, 3 \mathrm{H}), 4.43 \sim 4.45(\mathrm{~m}, 1 \mathrm{H}), 7.26 \sim 7.29(\mathrm{~m}, 2 \mathrm{H}), 7.76 \sim$ $7.78(\mathrm{~m}, 2 \mathrm{H}) ;{ }^{13} \mathrm{C} \mathrm{NMR}\left(75 \mathrm{MHz}, \mathrm{CDCl}_{3}\right) \delta: 21.6,30.3$, 54.6, 127.1, 129.6, 140.7, 142.9; MS (EI) $m / z: 227\left(\mathrm{M}^{+}\right)$.

Supporting Information ${ }^{1} \mathrm{H}$ NMR and ${ }^{13} \mathrm{C}$ NMR spectra for compounds 3aa $\sim \mathbf{3}$ an and 3ba $\sim$ 3la. The Supporting Information is available free of charge via the Internet at http://sioc-journal.cn/.

\section{References}

[1] For recent reviews, see:

(a) Studer, A.; Curran, D. P. Angew. Chem. Int. Ed. 2016, 55, 58. (b) Yan, M.; Lo, J. C.; J. Edwards, T.; Baran, P. S. J. Am. Chem. Soc. 2016, 138, 12692.

(c) Kärkäs, M. D. ACS Catal. 2017, 7, 4999.

(d) Jiang,Y.; Xu, K.; Zeng, C. C. Chem. Rev. 2018, 118, 4485.

[2] For recent reviews, see:

(a) McCann, S. D.; Stahl, S. S. Acc. Chem. Res. 2015, 48, 1756.

(b) Shimkin, K. W.; Watson, B. D. A. J. Org. Chem. 2015, 11, 2278.

(c) Guo, X.-X.; Gu, D.-W.; Wu, Z.; Zhang, W. B. Chem. Rev. 2015, 115, 1622 .

(d) Liang, Y.-F.; Jiao, N. Acc. Chem. Res. 2017, 50, 1640.

[3] For recent reviews, see:

(a) Zhu, X.; Chiba, S. Chem. Soc. Rev. 2016, 45, 4504.

(b) Tang, X. D.; Wu, W. Q.; Zeng, W.; Jiang, H. F. Acc. Chem. Res. 2018, 51, 1092.

[4] For recent examples, see:

(a) Lee, C.; Wang, X.; Jang, H.-Y. Org. Lett. 2015, 17, 1130,

(b) Chen, C.-Y.; Tang, G.; He, F.; Wang, Z.; Jing, H.; Faessler, R. Org. Lett. 2016, 18, 1690.

(c) Ryan, M. C.; Martinelli, J. R.; Stahl, S. S. J. Am. Chem. Soc. 2018, 140, 9074

[5] (a) Talley, J. J.; Brown, D. L.; Carter, J. S.; Graneto, M. J.; Koboldt, C. M.; Masferrer, J. L.; Perkins, W. E.; Rogers, R. S.; Shaffer, A. F.; Zhang, Y. Y.; Zweifel, B. S.; Seibert, K. J. Med. Chem. 2000, 43, 775

(b) Shen, C.-H.; Wang, Y.-F.; Kovalevsky, A. Y.; Harrison, R. W.; Weber, I. T. FEBS J. 2010, 277, 3699.

(c) McCormack, P. L. Durgs 2011, 71, 2457.

[6] (a) De Boer, T. J.; Backer, H. J. Org. Synth. 1954, 34, 96. (b) Harmata, M.; Zheng, P.; Huang, C.; Gomes, M. G.; Ying, W.; Ranyanil, K.-O.; Balan, G.; Calkins, N. L. J. Org. Chem. 2006, 72, 683 . 
[7] (a) Yin, J.; Buchwald, S. L. J. Am. Chem. Soc. 2002, 124, 6043

(b) Burton, G.; Cao, P.; Li, G.; Rivero, R. Org. Lett. 2003, 5, 4373.

(c) Rosen, B. R.; Ruble, J. C.; Beauchamp, T. J.; Navarro, A. Org. Lett. 2011, 13, 2564.

(d) Baffoe, J.; Hoe, M. Y.; Toure, B. B. Org. Lett. 2010, 12, 1532

(e) Audisio, D.; Messaoudi, S.; Peyrat, J. F.; Brion, J. D.; Alami, M. J. Org. Chem. 2011, 76, 4995.

[8] (a) Shekhar, S.; Dunn, T. B.; Kotecki, B. J.; Montavon, D. K.; Cullen, S. C. J. Org. Chem. 2011, 76, 4552.

(b) Moon, S.-Y.; Nam, J.; Rathwell, K.; Kim, W.-S. Org. Lett. 2014, 16,338 .

[9] (a) Shi, F.; Tse, M. K.; Zhou, S.; Pohl, M.-M.; Radnik, J.; Hubner, S.; Jähnisch, K.; Brückner, A.; Beller, M. J. Am. Chem. Soc. 2009, 131,1775 .

(b) Zhu, M.; Fujita, K.; Yamaguchi, R. Org. Lett. 2010, 12, 1336.

(c) Cui, X.; Shi, F.; Zhang, Y.; Deng, Y. Tetrahedron Lett. 2010, 51, 2048 .

(d) Watson, A. J. A.; Maxwell, A. C.; Williams, J. M. J. J. Org. Chem. 2011, 76, 2328.

(e) Shekhar, S.; Dunn, T. B.; Kotecki, B. J.; Montavon, D. K.; Cullen, S. C. J. Org. Chem. 2011, 76, 4552.

[10] Tang, X. D.; Huang, L. B.; Qi, C. R.; Wu, X.; Wu, W. Q.; Jiang, H. F. Chem. Commun. 2013, 49, 6102.

[11] Huang, X.; Wang, J. C.; Ni, Z. Q.; Wang, S. C.; Pan, Y. J. Chem. Commun. 2014, 50, 4582 .

[12] Zhu, M.; Wei, W.; Liu, C. L.; Yang, D. S.; Wen, J. W.; You, J. M.; Wang, H. Adv. Synth. Catal. 2015, 357, 987.

[13] Pan, X. J.; Gao, J.; Liu, J.; Lai, J. Y.; Jiang, H. F.; Yuan, G. Q. Green Chem. 2015, 17, 1400.

[14] Yang, K.; Ke, M. L.; Lin, Y. G.; Song, Q. L. Green Chem. 2015, 17, 1395.

[15] (a) Zhu, H.; Shen, Y.; Deng, Q.; Tu, T. Chem. Commun. 2015, 51, 16573.

(b) Zhang, F.; Zheng, D.; Lai, L.; Cheng, J.; Sun, J.; Wu, J. Org. Lett. 2018, 20, 1167.
[16] (a) Yotphan, S.; Sumunnee, L.; Beukeaw, D.; Buathongjan, C.; Reutrakul, V. Org. Biomol. Chem. 2016, 14, 590.

(b) Feng, J.-B.; Wu, X.-F. Org. Biomol. Chem. 2016, 14, 6951.

(c) Parumala, S. K. R.; Peddinti, R. K. Tetrahedron Lett. 2016, 57, 1232.

(d) Zhu, M.; Wei, W.; Yang, D.; Cui, H.; Wang, L.; Meng, G.; Wang, H. Org. Biomol. Chem. 2017, 15, 4789.

(e) Yu, H.; Zhang, Y. Chin. J. Chem. 2016, 34, 359

[17] (a) Powell, D. A.; Fan, H. J. Org. Chem. 2010, 75, 2726.

(b) Li, W.; Beller, M.; Wu, X.-F. Chem. Commun. 2014, 50, 9513.

(c) Ji, J.; Liu, Z.; Liu, P.; Sun, P. P. Org. Biomol. Chem. 2016, 14 7018 .

(d) Zhang, W.; Luo, M. Chem. Commun. 2016, 52, 2980.

(e) Shyam, P. K.; Jang, H.-Y. J. Org. Chem. 2017, 82, 1761

[18] (a) Yang, F.-L.; Tian, S.-K. Tetrahedron Lett. 2017, 58, 487. (b) Chung, S.; Kim, J. Tetrahedron Lett. 2019, 60, 792.

[19] (a) Li, L.; Li, Z.-L.; Gu, Q.-S.; Wang, N.; Liu, X.-Y. Sci. Adv. 2017, 3, e1701487.

(b) Li, X.-T.; Gu, Q.-S.; Dong, X.-Y.; Meng, X.; Liu,X.-Y. Angew. Chem. 2018, 57, 7668.

(c) Lin, J.-S.; Li, T.-T.; Liu, J.-R.; Jiao, G.-Y.; Gu, Q.-S.; Cheng, J.-T.; Guo, Y.-L.; Hong, X.; Liu, X.-Y. J. Am. Chem. Soc. 2019 , 141, 1074.

[20] Intermediate 5 has been proposed by some previous reports, see: (a) Yang, F.-L.; Tian, S.-K. Tetrahedron Lett. 2017, 58, 487.

(b) Li, X. Q.; Xu, X. S.; Hu, P. Z.; Xiao, X. Q.; Zhou, C. J. Org. Chem. 2013, 78, 7343.

(c) Yang, F.-L.; Tian, S.-K. Angew. Chem., Int. Ed. 2013, 52, 4929.

[21] The sulfonyl radical could be initiated by $\mathrm{O}_{2}$, for selected examples, see:

(a) Lu, Q.; Zhang, J.; Zhao, G.; Qi, Y.; Wang, H.; Lei, A. J. Am Chem. Soc. 2013, 135, 11481.

(b) Shen, T.; Yuan, Y.; Song, S.; Jiao, N. Chem. Commun. 2014, 50, 4115 .

[22] Alonso, E.; Ramón, D. J.; Yus, M. Tetrahedron 1997, 53, 14355

(Li, L.; Fan, Y.) 